\title{
A Systematic Classification and Labelling Approach to Support a Circular Economy Ecosystem for NdFeB-Type
}

\section{Magnet}

\author{
Carlo Burkhardt ${ }^{1}$, Antje Lehmann ${ }^{1}$, Benjamin Podmiljsak ${ }^{2}$ and Spomenka Kobe ${ }^{2}$ \\ 1. Institute for Precious and Technology Metals, Pforzheim University, Pforzheim 75175, Germany \\ 2. Department of Nanostructured Materials, Institut Jozef-Stefan, Ljubljana 1000, Slovenia
}

\begin{abstract}
Availability of magnetic materials is most crucial for modern Europe, as they are integral to energy conversion across the renewable energy and electric mobility sectors. Unfortunately, there is still no circular economy to reuse and capture value for these types of materials. With the prediction that the need for NdFeB Rare Earth (RE) magnets will double in the next 10 years, this problem becomes even more urgent. As the quality of the recollected materials varies significantly, the development of a classification system for recyclate grades of EOL NdFeB magnets in combination with an eco-labelling system for newly produced $\mathrm{RE}$ permanent magnets is proposed to clearly identify different magnet types and qualities. It categorises the NdFeB magnets by technical pre-processing requirements, facilitating use of the highly effective HPMS process (Hydrogen Processing of Magnetic Scrap) for re-processing extracted materials directly from NdFeB alloy. The proposed measures will have a great impact to overcome existing low recycling rates due to poor collection, high leakages of collected materials into non-suitable channels, and inappropriate interface management between logistics, mechanical pre-processing and metallurgical metals recovery.
\end{abstract}

Key words: NdFeB permanent magnet, magnet recycling, HPMS, hydrogen decrepitation, labelling \& grading, data matrix code (DMC), reycling factor, MaXycle, SUSMAGPRO.

\section{Introduction}

NdFeB Rare Earth (RE) magnets play a fundamental role in the shift to a clean energy future [1-4]. They are used in wind turbine generators, in electric vehicles (EV) and across other sectors, underpinning an industry worth more than $\$ 1$ trillion worldwide (2015) [5]. Driven by increased adoption of EV and green technologies, the global demand for NdFeB magnets ( 80,000 t/a 2017) is predicted to double in 2020 [6] and could grow at $15-20 \%$ p.a. for the next decade [5]. The ability of European companies to participate fully in these markets is restricted by the near-monopoly supply of RE in China and current trade conflicts, increases price

Corresponding author: Carlo Burkhardt, Ph.D., professor, research fields: REE recycling, sinter-based additive manufacturing, material characterisation. volatility and vulnerability of the market even further. Added to this, currently less than $1 \%$ of the world's REs are being recycled [7]. As the EU has no large, easily exploitable deposits of REs, its member states import $~ 99 \%$ of their NdFeB magnets, only 1,000 t/a are produced here [7]. Advanced research and innovation to improve exploitation as a secondary resource is therefore needed, with the recycling potential for RE magnets worldwide estimated as being in the range 14,000-20,000 t/a by $2020[8,9]$.

To recycle end-of-life magnets from waste of electric and electronic equipment (WEEE) and End-Of-Life vehicles, devices must be separated from waste streams and magnets liberated from components. As currently most electronics are shredded, recycling by associated chemical and pyrometallurgical routes is expensive, energy intensive [2] and still far from optimal and the vision of an efficient "circular economy". 
The highly effective HPMS process (Hydrogen Processing of Magnet Scrap) [10] to re-process extracted materials directly from the NdFeB alloy is a very promising alternative. With HPMS, NdFeB magnets break down into a friable powder, which can be separated mechanically from remaining impurities [11-13], creating much more environmentally friendly "short cycle" re-processing routes.

However, several challenges hinder remanufacturing from unknown waste sources via HPMS:

- identification of different types of scrap magnets and levels of contamination;

- measures to deal with impurities in waste streams;

- fluctuations in properties of recycled materials of varying compositions;

- recycling “unfriendly” designs.

The work carried out in this investigation is laying the foundation for the establishment of a future labelling system for hard magnets and the development of a unified classification system for End-Of-Life NdFeB magnet (EOLM), allowing recommendations to original equipment manufacturers (OEM) about recycling-friendly design and manufacturing for maximum recyclability.

The generated classification system will not only help the circular economy on recycling but also raise awareness of how cost-effective any magnet may be recycled, thus providing valuable information to

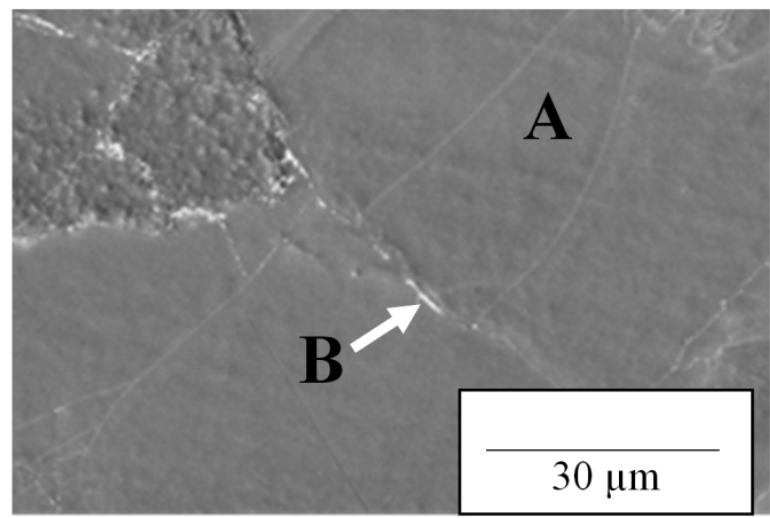

(a) end-users and decision makers about environmental impacts, helping to facilitate efficient eco-design.

\section{Experimental Setup}

In a systematic approach [14, 15], EOLM in different conditions is analysed to subsequently classify the magnets for recyclability with HPMS in a standardised grading system with the help of optical microscopy, HR-SEM, EDS, ICP-OES and oxygen analysis.

\section{Experimental Results}

\subsection{Chemical Composition and Microstructure}

EOLM from various scrap sources (production scrap, traction motors, wind generators, audiomodules and loudspeakers) in various conditions was analysed for classification, as illustrated in Fig. 1.

As suspected, it was found that the NdFeB-type magnets from the various scrap sources vary significantly in their chemical composition. This is on one hand attributed to their magnet and temperature grade (e.g. N30 vs. N52 SH), on the other hand, there are various other factors that influence the magnetic composition. As most OEM magnet users do not specify the chemical composition but the magnet/temperature grade of their product, significant differences in chemical compositions were found in the Nd, Pr, Dy and $\mathrm{Tb}$ content, which are attributed to raw material

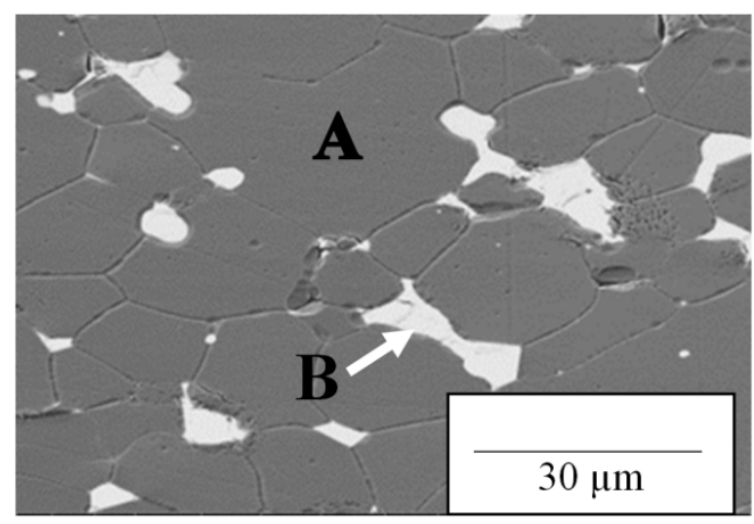

(b)

Fig. 1 Typical microstructure of EOL magnets: (a) EOLM from electric motor of DIY drilling machine; (b) EOLM from wind turbine generator ( $\mathrm{A}: \mathrm{Nd}_{2} \mathrm{Fe}_{14} \mathrm{~B}$ phase, $\mathrm{B}$ : Nd-rich phase). 
supply from different mines and/or different manufacturing strategies of the respective magnet producers. Similar variations could be found for alloying additions such as Ga, Zr and Co. Additionally, variations in the grain size and amount of Nd-rich grain boundary phase were examined, again attributed to different manufacturing strategies and/or magnet grade/quality.

\subsection{Corrosion}

In a determination of the elements oxygen, nitrogen and hydrogen by intert gas fusion (ONH analysis), significant differences were also found in the oxygen content of the EOLM. Whereas in production scrap very low oxygen contents of $<350$ ppm were measured, in other EOL magnets (e.g. from traction motors and audiomodules from smart phones) $\mathrm{O}_{2}$-values of $>4,000 \mathrm{ppm}$ could be found. Based on these investigations, the factors influencing the oxygen content of EOLM are operating conditions, coatings and service life; with shorter operating life and/or denser coating layer leading to smaller $\mathrm{O}_{2}$-values. The operating conditions however are suspected to be the main influencing factor, with more corrosive environment resulting in higher $\mathrm{O}_{2}$-values.

\subsection{Coatings}

Because of the extreme reactivity of $\mathrm{Nd}$ to oxygen, NdFeB-type magnets for serial applications must be coated to ensure acceptable service life [16]. In this investigation, a large variety of coating layers of EOLM were analysed with optical microscopy and SEM/EDS; ranging from e.g. electroplated single-layer $\mathrm{Ni}$ or $\mathrm{Zn}$ coatings over electroplated multi-layer coatings (e.g. Ni-Cu-Ni) to single- or multi-layer polymer coatings with or without embedded fibrous material. In traction motors, also uncoated EOLMs, treated with Dy-grain boundary diffusion processes [17] were found.

Fig. 2 illustrates different typical coating types, as found in $\mathrm{EOL} \mathrm{NdFeB}$ magnets for various applications.

\subsection{Synopsis of the Analysis of EOLM from Various} Scrap Sources

End-of-Life magnets come in a wide variety. Not only with respect to the initial magnet type (Sm-Co, Ferrite, AlNiCo or NdFeB), but even when narrowing down the analysis to NdFeB-type material only, a wide variety of initial chemical compositions can be found. At the end of their service life, EOLM will vary in their state of corrosion, and their coating type, with currently no systematic pattern discernible, as even for the same application and nominally same magnet grade, very different coating types (e.g. Ni, Zn, multilayer and polymer) applied by various processes (electroplating, spray-coating etc.) could be found. It is suspected that the type of coating for a certain application is more or less arbitrarily, resulting from the production capabilities and the available equipment of the respective manufacturer.

\subsection{Consequences of the Variety of NdFeB-Type Magnets for HPMS EOLM Recycling}

The HPMS process is a very efficient short-cut recycling method where on exposure to hydrogen the sintered NdFeB magnets break down into a friable, demagnetised, hydrogenated powder containing an interstitial hydride of $\mathrm{Nd}_{2} \mathrm{Fe}_{14} \mathrm{BH}_{x}$ (10 microns) and smaller particles $(<\quad 1 \quad$ micron $)$ from the grain-boundary phase $\left(\mathrm{NdH}_{2.7}\right)$, which can be separated mechanically from the coatings, glues, screws and fixtures. It is then possible to re-process the extracted materials directly from the Nd-Fe-B alloy, creating "short-cycle” reprocessing routes (see Fig. 3) that are more effective in terms of materials recovery and increase the yield compared to primary production by $25 \%$ as the material stays as an alloy all the way through the recycling processes.

The separated powders/magnets can then be re-manufactured into sintered magnets (Route A), polymer bonded magnets (Route B) and metal-injection-moulded magnets (Route C) and cast alloys (Route D) [12-15]. 

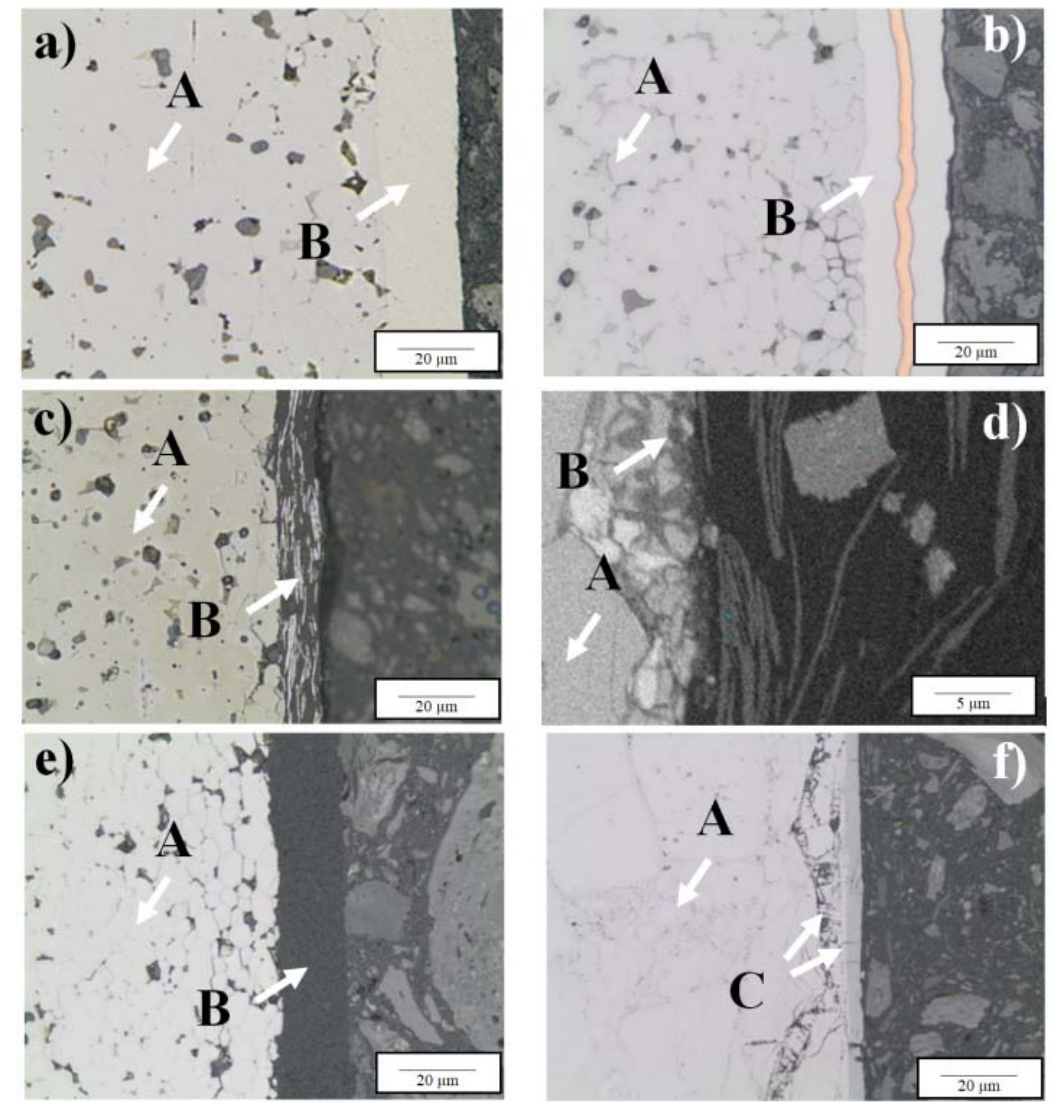

Fig. 2 Different typical coatings of EOLM: (a) Ni (electroplated); (b) multilayer Ni-Cu-Ni (electroplated); (c) and (d) polymer with fibres (different magnifications); (e) single layer polymer; (f) Dy-grain boundary diffusion. (“A” always illustrating the magnet, “ $B$ ” the coating, “ $C$ ” Dy-grain boundary diffusion).

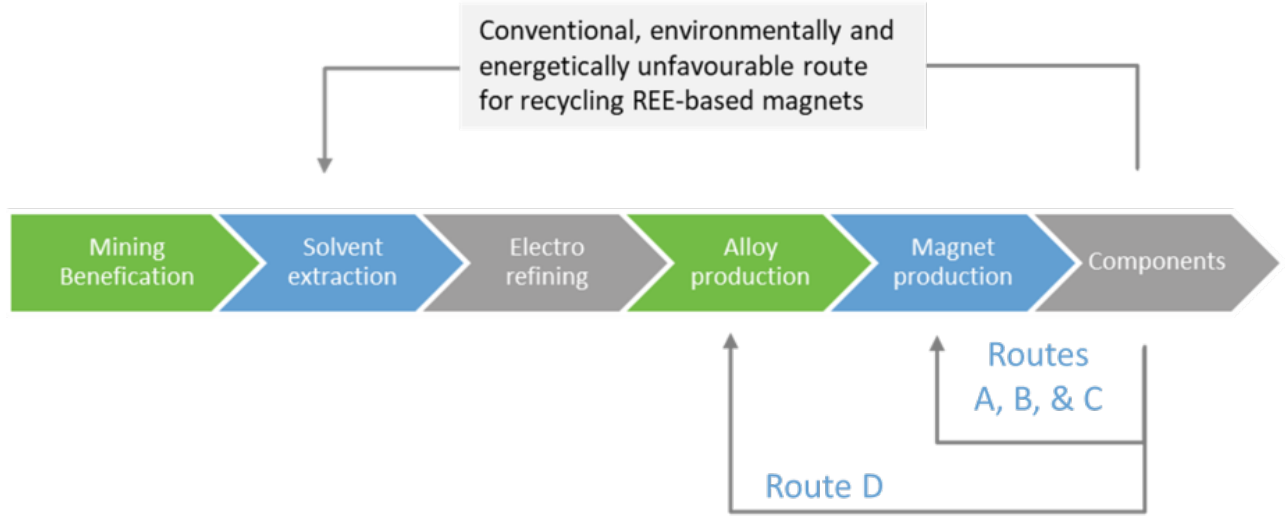

Fig. 3 Conventional production route for Nd-Fe-B magnets and the HPMS “short-cycle” route [17].

However, as seen from the analysis of EOLM from various scrap sources carried out for this work, there are several critical challenges to be addressed when HPMS remanufacturing the extracted REE (Rare Earth Element) alloys with undefined origin.

The varying chemical compositions could lead to fluctuations in the properties of the recycled materials, and impurities in waste streams from the coating materials or from the higher oxygen content of the starting sintered magnets are also potentially detrimental to the process, as a high oxygen content results in the oxidation of the Nd-rich phase, with a consequent loss of the liquid phase for the sintering process, as shown by other work [18]. By adding additional $\mathrm{Nd}$ in the 
form of hydrides or blending the recyclate with overstochiometric NdFeB-type material, the oxidation can be compensated [19], however adding complexity to the process.

Some of the coatings are not permeable to the hydrogen, so they must be punctured to initiate the hydrogen decrepitation (HD) reaction. Other coatings (e.g. some polymers) break into small pieces during processing, which make them very difficult to separate from the magnetic powder after HPMS, resulting in contaminated material unsuitable for re-processing. Electroplated Ni-coatings on the other hand flake off in large pieces $(>100 \mu \mathrm{m})$ during the HPMS process and can be easily sieved off in a cost-effective after-treatment.

\subsection{Magnet Accessibility and Fixation Methods}

Whereas with production scrap and in some assemblies the EOLM is easily accessible, other assemblies are not designed in a recycling friendly manner: their components are glued or held together by screws, which can be severely corroded over life-time. In such cases, a tailored disassembly concept of the component may be a severe cost factor in large-scale HPMS recycling. The same is true for the fixation of the EOLM, where everything from mechanical fixation up to complete coverage of the magnet in multi-component glue was found. Again, disassembly of such magnets may be a severe cost factor in large-scale HPMS recycling.

\section{Systematic Classification and Labelling Approach}

As seen above, depending on their origin, purpose, place of installation and operational life time, the qualities of recollected magnets vary significantly, especially of alloying constituents, state of corrosion, coatings and other residues. As these differences influence the quality, process-complexity and process-stability for recycled magnets [16, 20], innovation must be embedded into broader contexts including regulatory innovations, to enable efficient and competitive recycling.

\subsection{Development of an HPMS Recycling Factor for EOLM}

In the medium term, the recycling-friendly design of NdFeB applications must be rewarded. However, future measures will have no effect on the material currently available. To enable the recycling of at least some existing EOLM lots, an attempt has been made to develop a recycling factor that will quickly indicate whether HPMS recycling a certain batch of EOLM magnets is economically attractive to a recycling company or not.

This recycling factor is calculated from various attributes that are either favourable or detrimental to the HPMS process. Each of these attributes has been linked to a digit, a low value representing "detrimental" and a high value "favourable". Attributes or properties that make HPMS recycling impossible have been valued zero (e.g. polymer bonded magnets or certain multi-layer polymer coatings). In a holistic approach, the different attributes were then weighted, and the assigned numerical values multiplied with each other.

The result is a number indicating whether a batch of EOLM can be easily recycled using the HPMS process. If this recycling factor is zero, the lot is not suitable for HPMS-processing.

The following attributes were employed to determine the recycling factor:

- production method (sintered, polymer-bonded or melt-spun MQ3);

- content of heavy REE (in w\%);

- type of coating (e.g. Ni, Zn, polymer; single or multi-layer);

- fixation (e.g. mechanical, glued);

- accessibility (ease of dismantling of the component to access the magnet);

- state of corrosion (mean oxygen content of the lot in $\mathrm{ppm})$;

- quantity (in tons resp. tons p.a.). 
Example Magnet 1

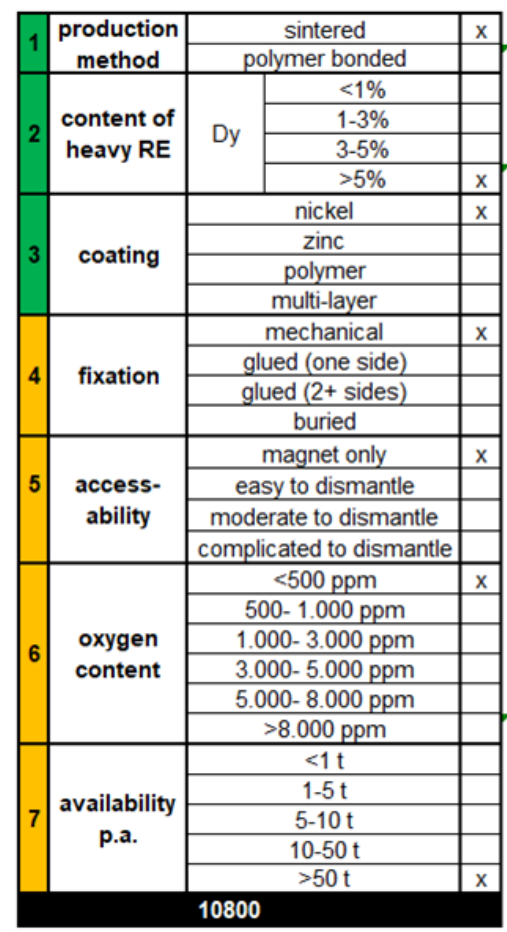

Example Magnet 2

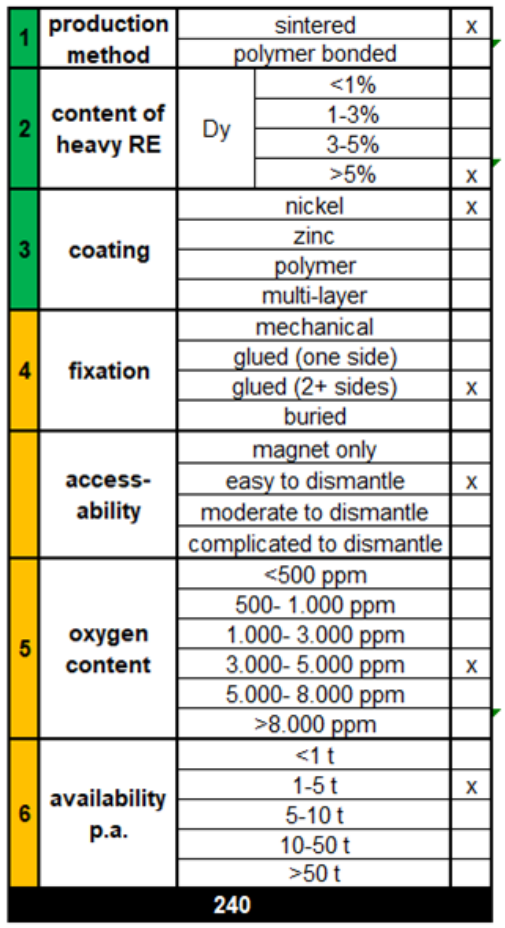

Example Magnet 3

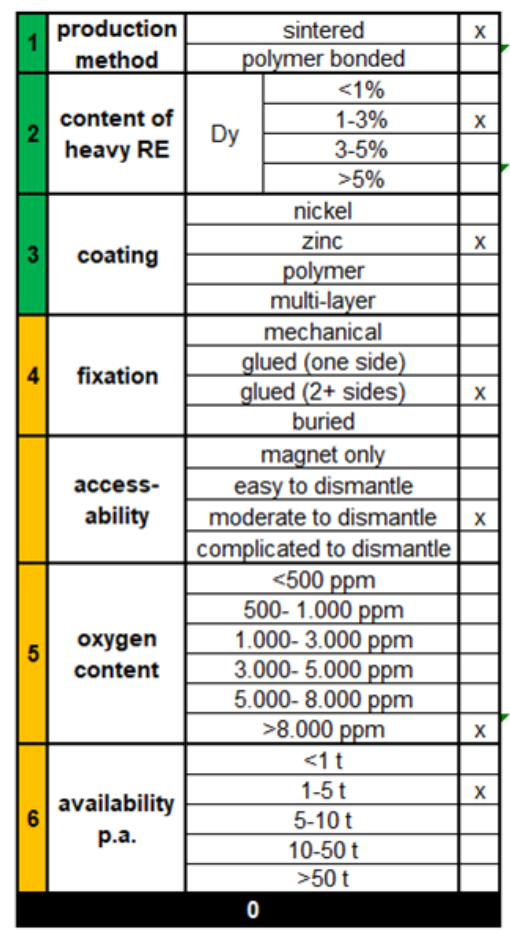

Fig. 4 HPMS recycling factors for different lots of sintered NdFeB-type magnets.

Fig. 4 illustrates the generated recycling factor (white number in black bottom line of the respective table) for three different recycling lots of sintered NdFeB-type magnets.

From the high recycling factor of 10,800 points can be seen that the batch of Example Magnet 1-which is $\mathrm{Ni}$-coated, having high Dy- and low $\mathrm{O}_{2}$-content, while being available as "magnet only" (not requiring disassembly) in a quantity > 50 tons-is highly attractive for HPMS recycling.

The lot of Example Magnet 2, being of same composition and having similar coating as that of Example Magnet 1, is much less attractive for recycling, indicated by a recycling factor of only 240 points. Albeit being easy to disassemble, these magnets are glued to the component, requiring a tailored disassembly concept for removal. The development of such process steps, always adapted to the application, is complex and since the available batch is relatively small (1-5 tons), there is a high risk of not covering costs here. The high oxygen content in the batch further reduces the attractiveness for recycling.

With Example Magnet 3, the high oxygen content of $>8.000 \mathrm{ppm}$ is responsible for a recycling factor of 0 , indicating that the heavily corroded batch is not suitable for HPMS processing.

\subsection{Feasibility of an HPMS Recycling Factor for EOLM}

The proposed recycling factor gives a relatively quick indication if a batch of EOLM is suitable for HPMS recycling. It has however, severe limitations: the determination of the content of heavy REE and the $\mathrm{O}_{2}$-content requires ICP-OES (inductively coupled plasma optical emission spectrometry) and $\mathrm{ONH}$ analysis (determination of the elements $\mathrm{O}, \mathrm{N}$, and $\mathrm{H}$ by inert gas fusion), which are costly and time-consuming. Furthermore, representative values can only be achieved if the batch is of uniform characteristics or an adequate number of samples are investigated.

Additionally, it may not be enough to consider the content of heavy REE only. Because of e.g. the 
increased reactivity of $\mathrm{Pr}$ with respect to hydrogen in the HPMS treatment or the effect of Co and Dy on the processing parameters when making HDDR powders of the recycled material, knowledge of the whole chemical composition of the magnets batch in question would be very beneficial.

Taking these facts into account, the introduction of an HPMS recycling factor must be accompanied by other measures to ensure effective and economically attractive $\mathrm{NdFeB}$ recycling.

\subsection{Labelling of NdFeB Magnets}

To achieve recycling rates in $\mathrm{NdFeB}$ magnets that are in the range of other technology metals, automated processing is required. For initial sorting and further downstream processing, labelling of magnetic components is therefore without alternative.

The initial information of the marking must be magnet type and production method, to allow initial sorting of NdFeB-, SmCo-, AlNiCo- and Ferrite magnets and to distinguish between e.g. sintered and polymer bonded magnets.

The second information must be the magnet grade, to separate high quality from low quality magnets and to bundle matching magnet qualities in larger batches. Here a specification of the chemical composition as exact as possible would be desirable - at least the content of heavy rare earths must be specified. Aware of the difficulties of protecting the know-how of manufacturer-specific formulations, the use of blockchain technology is currently being examined to find the best possible compromise between the differing interests of manufacturers and recyclers.

Another information vital is the coating type, again to separate magnets with different coatings and to bundle in larger batches for processing (e.g. coating removal or other pre-HPMS treatment).

Other information in the marking could contain a certified magnets producer code and other specifics which would help to eliminate illegal mining and/or manufacturing.

\subsection{Labelling Methods for Magnetic Products}

For the marking itself, both surface and subsurface labelling were considered. Subsurface labelling in form of tracers in the matrix material, as e.g. used in sorting plastic waste [21] or for the traceability of firearms, proved to be unsuitable, as it rules out multiple recycling (mix-up of tracers) and is accompanied with increased production costs.

Surface markings, on the other hand, have been widely used for many years in a variety of industrial applications in the form of barcodes and QR codes.

In many applications (e.g. traceability in automotive production, labelling of electronic components), so-called DMC codes (Digital Matrix Codes) have recently gained acceptance. A comparatively large amount of information can be stored in the form of alphanumeric codes in the smallest of spaces. DMC codes are also characterized by a high error tolerance and can be machine-read very quickly.

In this work, the magnet labelling information described above was translated into a 16-digit alphanumerical code, which allows to generate a $16 \times$ 16 pixel DMC with a minimum size of $3.4 \times 3.4 \mathrm{~mm}$. Such code can be ink-jetted or laser-engraved onto the coating of an $\mathrm{NdFeB}$ magnet with a variety of available machines and read by standard DMC readers or even free smartphone apps. The DMC is small enough to be printed onto the vast majority of recyclable magnets in automotive and power generation purposes, which are suspected to be the main material sources for future recycling. If appropriate measures are taken, full traceability can also be ensured by labelling of the assembly or the magnet's material certificate only.

The different labelling approaches are currently discussed with selected OEMs in various fields of application. Intensive cooperation with working groups dealing with the recycling of vehicle batteries will help to ensure that a feasible, transferable and environmentally sound solution can be developed, meeting the needs and standards of large-scale manufacturing. 


\begin{tabular}{|c|c|c|c|c|}
\hline $\begin{array}{c}\text { Type of Magnet } \\
\text { select from drop down list }\end{array}$ & $\begin{array}{c}\text { Type of Coating } \\
\text { select from drop down list }\end{array}$ & $\begin{array}{c}\text { Heavy RE-Content [\%] } \\
\text { enter value in mass } \%\end{array}$ & $\begin{array}{c}\text { Magnet Grade } \\
\text { select from drop down list }\end{array}$ & $\begin{array}{c}\text { Certified Producer Code } \\
\text { select from drop down list }\end{array}$ \\
\hline NdFeB_sintered & $\mathrm{Zn}$ & 0,30 & N27 $\mathrm{EH}$ & Magneti \\
\hline
\end{tabular}

Fig. 5 MaXycle code-generator (left) and resulting DMC-code (right).

In the MaXycle project, an automated code-generator was programmed and made available in open access for test purposes, as illustrated in Fig. 5.

The information contained in the label is giving vital information needed for the determination of the recycling factor by giving the parameters "production method", "content of heavy RE" and "coating” (green rows 1, 2 and 3 in Fig. 4).

If OEMs would define a minimum recycling factor for a future NdFeB application already in the design phase, recycling-friendly design would be encouraged, still giving the magnets and/or components producer a substantial degree of freedom in how to achieve the factor (e.g. by easy access to the magnet or phasing out certain coating methods, etc.)

\section{Conclusion}

As the EU has no large, easily exploitable deposits of REEs, recycling of NdFeB type materials is not an option, it is a must. Advanced research and innovation to improve exploitation as a secondary resource is therefore needed, with the HPMS process being a very efficient short-cut recycling method where the extracted materials can be re-processed directly from the Nd-Fe-B alloy, creating "short-cycle" reprocessing routes.

To be commercially successful, several critical challenges need to be addressed, e.g. measures how to deal with the varying chemical compositions and impurities in the waste stream and the introduction of recycling-friendly designs, facilitating access to the magnet, using recycling friendly fixation and coating methods.

In a systematic approach, a recycling factor for $\mathrm{NdFeB}$ magnets in currently existing waste streams was generated, giving a relatively quick indication if a batch of EOLM is suitable for HPMS recycling. To ensure effective and economically attractive $\mathrm{NdFeB}$ recycling, the introduction of an HPMS recycling factor must be accompanied by a concise and comprehensive labelling of new magnets for future applications.

Such labelling was created in form of a data matrix code (DMC), with full traceability being ensured either by labelling the magnet, the assembly or the magnet's material certificate. Labelling in combination with the definition of minimum recycling factors for future NdFeB magnets application would encourage recycling-friendly design.

To create a solid solution for grading and labeling, which will be widely accepted by industry and other stakeholders, substantial further work is necessary and will be carried out in the EU-funded projects MaXycle and SUSMAGPRO.

\section{Acknowledgments}

SUSMAGPRO has received funding from the European Union's Horizon 2020 research and innovation programme under grant agreement No. 821114, MaXycle has received funding from the ERA-MIN2 research \& innovation programme on raw materials to foster circular economy.

\section{References}

[1] European Commission. "Critical Raw Materials.” http://ec.europa.eu/growth/sectors/raw-materi als/specific-interest/critical_en.

[2] Sprecher, B., Xiao, Y., Walton, A., Speight, J. D., Harris, I. R., Kleijn, R., Visser, G., and Kramer, G. J. 2014. "Life Cycle Inventory of the Production of Rare Earths and the Subsequent Production of Nd-Fe-B Rare Earth Permanent Magnets.” Environmental Science and 
Technology 48: 3951-8.

[3] Constantinides, S. 2016. "Market Outlook for Ferrite, Rare Earth and Other Permanent Magnets.” Presented at International Forum on Magnetic Applications, Technologies \& Materials, Jacksonville, USA.

[4] International Energy Agency. 2016. Energy, Climate Change \& Environment: 2016 Insights.

[5] Kooroshy, J., Tiess, G., Tukker, A., and Walton, A. 2015. Strengthening the European Rare Earths Supply Chain: Challenges and Policy Options, ERECON Final Report. https://ec.europa.eu/growth/sectors/raw-materials/specific interest/erecon_en.

[6] Shaw, S., and Constantinides, S. 2012. "Permanent Magnets: The Demand for Rare Earths.” Presented at 8th International Rare Earths Conference, Hong Kong.

[7] Binnemans, K., et al. 2013. "Recycling of Rare Earths: A Critical Review.” Journal of Cleaner Production 51: 1-22. doi: 10.1016/j.jclepro.2012.12.037.

[8] Schulze, R., and Buchert, M. 2016. "Estimates of Global REE Recycling Potentials from NdFeB Magnet Material.” Resources, Conservation and Recycling 113: 12-27. doi: 10.1016/j.resconrec.2016.05.004.

[9] Sprecher, B., Kleijn, R., and Kramer, G. J. 2014. "Recycling Potential of Neodymium: The Case of Computer Hard Disk Drives.” Environmental Science and Technology 48: 9506-13. doi: 10.1021/es501572z.

[10] Walton, A., Williams, A. J., Speight, J. D., and Harris, I. R. 2014. Magnet recycling. US Patent No. US 13/169,839, priority 2010, application 2012, granted 2014.

[11] Walton, A., et al. 2015. "The Use of Hydrogen to Separate and Recycle Neodymium-Iron-Boron-type Magnets from Electronic Waste.” Journal of Cleaner Production 104: 236-41. doi: 10.1016/j.jclepro.2015.05.033.

[12] European Commission. "CORDIS EU Research Results, 2020, Rare Earth Magnet Recovery for Environmental and Resource Protection.” Accessed Sep. 20, 2020. https://cordis.europa.eu/project/id/ 310240.

[13] European Commission. "CORDIS EU Research Results,
2020; Resource Efficient Production Route for Rare Earth Magnets.” Accessed Sep. 20, 2020. https://cordis.europa.eu/project/id/636881.

[14] Podmiljsak, B., and Burkhardt, C. "A Novel Circular Economy for Sustainable RE-Based Magnets.” Accessed Sep. 20, 2020. http://.maxycle.eu/.

[15] European Commission. "CORDIS EU Research Results, 2020, Sustainable Recovery, Reprocessing and Reuse of Rare-Earth Magnets in a Circular Economy.” https://cordis. europa.eu/project/id/821114. Accessed Sep. 20, 2020. https://cordis.europa.eu/project/rcn/223261/factsheet/en.

[16] Li, Y., Evans, H. E., Harris, I. R., and Jones, I. 2003. "The Oxidation of NdFeB Magnets." Oxidation of Metals 59: $167-82$

[17] Suppan, G., Ruehrig, M., Kanitz, A., and Gores, H. J. 2015. "Electroplating Dysprosium from IL-Based Solutions: A Promising Electrochemical Step to Produce Stronger High Performance Nd(Dy)-Fe-B Sintered Magnets.” Journal of the Electrochemical Society 162 (8): 382-8.

[18] Herraiz, E., Bradshaw, A., Rowson, N. A., Onal, M. A. R., Franczak, A., Poenaru, I., and Walton, A. 2016. "Recycling of Nd-Fe-B Magnets by Hydrocyclone Separation and Re-sintering." In Proceedings of the 24th International Workshop on Rare Earth Permanent Magnets and Their Applications, Darmstadt, 498-503.

[19] Degri, M. J. 2014. "The Processing and Characterisation of Recycled NdFeB-Type Sintered Magnets.” PhD thesis, The University of Birmingham.

[20] Yang, Y., Walton, A., Sheridan, R., Güth, K., Gauß, R., et al. 2017. "REE Recovery from End-of-Life NdFeB Permanent Magnet Scrap: A Critical Review.” J. Sustain. Metall. 3: 122-49. doi 10.1007/s40831-016-0090-4.

[21] Woidasky, J., Heyde, M., Sander, I., Moesslein, J., Fahr, M., Richards, B., Turshatov, A., and Lang-Koetz, C. 2017. "Hochwertiges Recycling Durch Tracer-Nutzung." ReSource 30 (4): 24-8. 